\title{
A UTILIZAÇÃO DE UMA CURVA PONDERAL DE GESTANTES NORMAIS NO DIAGNOSTICO DE DESNUTRIÇÃO INTRA-UTERINA
}

\author{
Arnaldo Augusto Franco de Siqueira* \\ Ana Cristina d'Andretta Tanaka* \\ Cyro Ciari Júnior * \\ Pedro Augusto Marcondes de Almeida
}

RSBU-B/287

Siqueira, A. A. F. de et al. - A utilização de uma curva ponderal de gestantes normais no diagnóstico de desnutrição intra-uterina. Rev. Saúde públ., $\mathbf{S}$. Paulo, 9:495-506, 1975.

RESUMo: Com a finalidade de estudar a influência do estado nutricional materno sobre o peso do recém-nascido, foram aplicadas as "Curvas de Gestantes Normais" em 1.347 gestantes normais. A utilização destas curvas permitiu identificar o estado nutricional materno, dividindo as gestantes em estudo em três grupos, sendo: 243 gestantes magras ou subnutridas, 894 normais e 210 obesas. Foram comparados os pesos dos recém-nascidos de cada grupo de gestantes $e$ verificou-se que a incidencia de prematuridade foi duas vezes maior entre os recém-nascidos de mulheres magras, sendo a incidência de pós-termo o dobro nas gestantes obesas. Verificou-se ainda que os pequenos para idade gestacional tem uma maior incidência nas gestantes magras e também nas normais, cujos ganhos de pesos se apresentem abaixo da média da "Curva Ponderal de Gestantes Normais".

Unitermos: Peso ao nascer. Ganho de peso materno. Prematuridade, poss-termo.

\section{N T R O D U C A O}

Uma das grandes preocupações atuais em Saúde Materno-Infantil é a alta morbidade e mortalidade a que estấo sujeitos os recém-nascidos com peso inferior a $2.500 \mathrm{~g}$.

Estas crianças podem ser divididas em dois grupos: um, dos recém-nascidos de baixo peso em virtude de gestaçōes mais curtas, que são os prematuros, sendo o seu crescimento intra-uterino adequado; e o outro grupo que são os de recém-nascidos com baixo peso para sua idade gestacional, isto é, tiveram seu crescimento intra-uterino deficiente.
A importância do diagnóstico desses dois grupos de recém-nascidos se deve às deficiências que irão apresentar tanto na morbidade e mortalidade durante o período neonatal, como no prognóstico do seu crescimento e desenvolvimento futuros.

Assim é que os problemas de saúde que apresentam os prematuros decorrem quase exclusivamente do baixo peso ou da imaturidade de órgãos e aparelhos, com que nascem estas crianças. Por outro lado, os recém-nascidos pequenos para a idade gestacional, além dos problemas de baixo peso, imaturidade de órgãos e aparelhos,

- Do Departamento de Prática Médica em Saúde Pública da Faculdade de Saúde Pública da USP - Av. Dr. Arnaldo, 715 - São Paulo, SP - Brasil. 
SIQUEIRA, A. A. F. de et al. - A utilização de uma curva ponderal de gestantes normais no diagnóstico de desnutrição intra-uterina. Rev. Saúde públ., S. Paulo, 9:495-506, 1975.

apresentam também problemas metabólicos e maior risco de ocorrer distúrbios no seu desenvolvimento neuropsicomotor.

Existem diversas causas que influenciam no crescimento intra-uterino inadequado do recém-nascido como o fumo, a insuficiência placentária, a hipertensão materna, a altitude do lugar ou, como afirma Gruenwald 2, através de uma limitação da linha de suprimento fetal, representada pela mãe e placenta.

Finalmente, outra causa muito importante (e talvez a mais importante em paises em desenvolvimento) é a subnutrição materna.

Houve tempo em que o feto era considerado um parasita, retirando do organismo materno, fossem quais fossem as suas reservas, todos os nutrientes de que necessitasse.

Hoje sabe-se que o feto sofre as consequêencias de carências alimentares da gestante durante a gravidez e também, muito provavelmente, de déficits nutricionais presentes no período pré-concepcional.

Em vista das evidências sentiu-se a necessidade de criar métodos para o diagnóstico do estado nutricional da gestante.

É sabido que em termos individuais é difícil esse diagnóstico ${ }^{3} \mathrm{em}$ virtude das alteraçōes fisiológicas do organismo materno inerentes ao estado gravídico. Em termos da população de gestantes a tarefa torna-se mais difícil ainda.

A utilização de métodos bioquímicos para o diagnóstico do estado nutricional exige, além de recursos de capital, em termos de equipamentos altamente sofisticados, recursos humanos especializados. Tanto num como noutro aspecto os países em desenvolvimento estão, assim, praticamente impossibilitados de trabalhar nessa linha.

Em relação ao estudo de hábitos alimentares e da composição de dietas, além das dificuldades de aceitação dos inquéritos alimentares por parte da população, devemos considerar também a falta de pessoal técnico e o fato de que, no Brasil, os hábitos alimentares variam enormemente de uma região para outra.

Pode-se lançar mão, como salienta Jelliffe ${ }^{4}$, de estudos antropométricos para o diagnóstico do estado nutricional de uma população.

Assim é que a medida de peso dos recém-nascidos permite identificar a existência de desnutrição na população de gestantes. As tabelas de peso e estatura são importantes métodos para um diagnóstico inicial de desnutrição em crianças.

Em nosso meio, Ciari Jr. ${ }^{1}$ tem se preocupado com a elaboração de curvas ponderais de gestantes normais.

Em outro trabalho por nós publicado ${ }^{5}$ utilizando os registros clínicos de pré-natal e do recém-nascido de 1.347 gestantes de 3 serviços de pré-natal, foram elaboradas curvas de ganho de peso de gestantes, que identificam gestantes magras (ou subnutridas), normais e obesas, conforme apresentem ao longo da gestação, pesos abaixo, dentro ou acima de uma faixa de valores normais.

Neste trabalho, através de aplicação das curvas ponderais de gestantes normais, pretende-se correlacionar o estado nutricional materno com o grau de exposição à desnutrição intra-uterina e prematuridade dos recém-nascidos.

\section{MATERIAL E MÉTODOS}

Para o presente estudo, foram utilizados registros clínicos de ficha de pré-natal e peso do recém-nascido de 1.347 gestantes normais, selecionadas de três serviços de atenção pré-natal, assim distribuídas: 482 do Serviço Pré-Natal do Centro de Saúde Geraldo de Paula Souza da Faculdade de Saúde Pública da USP, 467 do Serviço de Pré-Natal da Escola Paulista de Medicina e 398 de uma clínica particular.

Para inclusão de cada caso no estudo foi usado o critério de normalidade durante a evolução da gravidez. Qualquer patologia apresentada no início, durante e no final da gestação excluía automaticamente a mulher do estudo. 
SIQUEIRA, A. A. F. de et al. - A utilização de uma curva ponderal de gestantes normais no diagnóstico de desnutricão intra-uterina. Rev. Saúde públ., S. Paulo, 9:495-506, 1975.

Por outro lado, as fichas que não apresentassem dados completos, como altura materna, idade gestacional, peso materno durante a gravidez e peso do recém-nascido, foram também excluídas. Os dados foram codificados e passados por cartões IBM. Foi elaborado um programa para a computação eletrônica dos dados (Linguagem Fortran IV, Cobol o Pack e o SPSS V5) *. Lma parte dos dados foram tabulados manualmente, elaborando-se com os resultados curvas de ganho de peso segundo a estatura da gestante.

Essas curras, elaboradas segundo metodologia descrita em outro trabalho ${ }^{5}$ permitem reconhecer as gestantes magras, normais e obesas.

Após a classificação de cada gestante com base nas curvas construídas, foram elaboradas tabelas relacionando peso do recém-nascido e idade gestacional para cada grupo de mulheres.

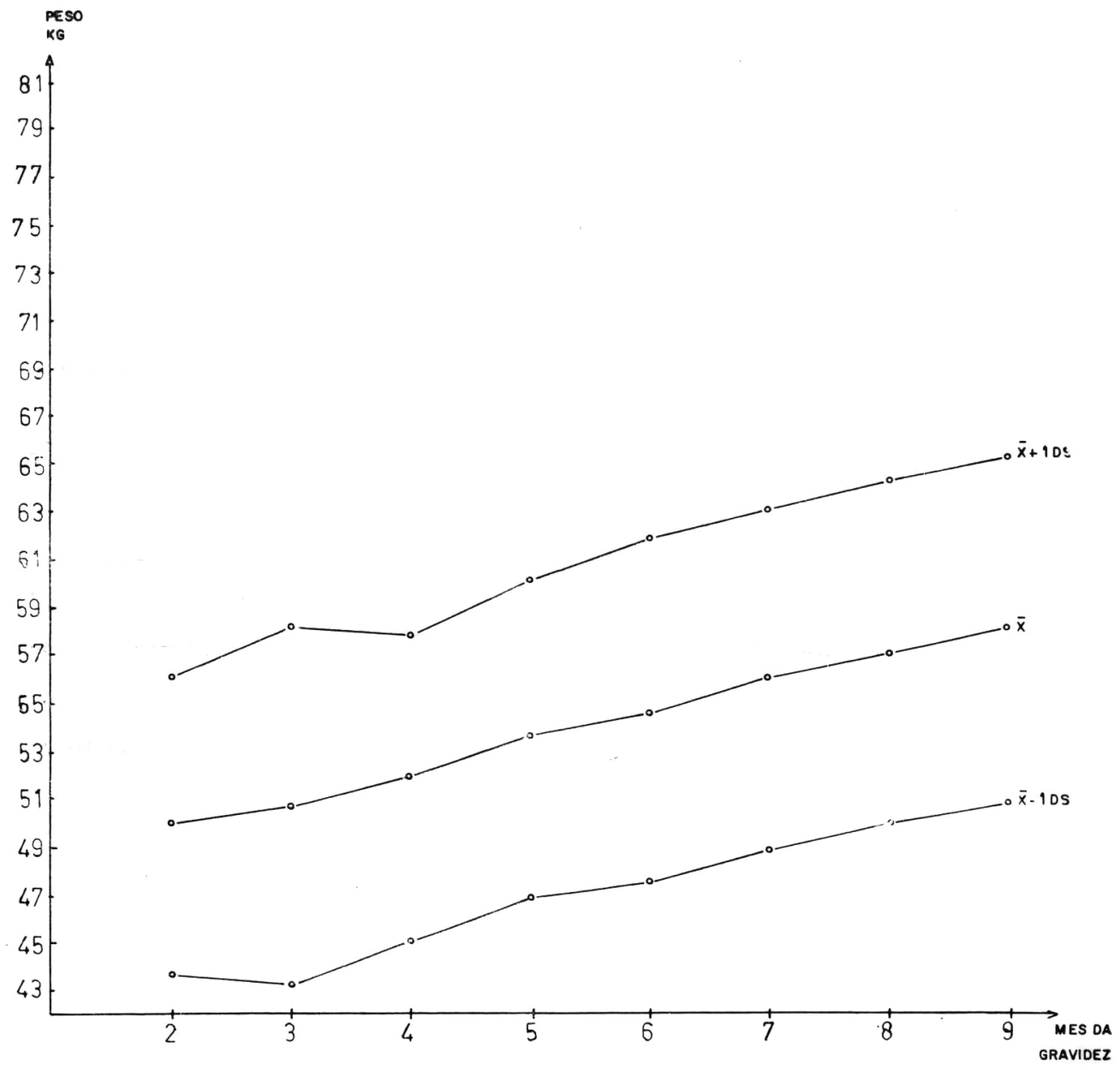

Fig. 1 - Curva ponderal para gestantes de $1,49 \mathrm{~m}$ e menos.

* O programa fol elaborado pelo Dr. Jair Lício Ferreira Santos e o Sr. Fernão Dias de Lima, do CEDIP da FSP/USP. 
SIQUEIRA, A. A. F. de et al. - A utilização de uma curva ponderal de gestantes normais no diagnóstico de desnutrição intra-uterina. Rev. Saúde puibl., S. Pauio, 9:495-506, 1975.

\section{RESULTADOS E DISCUSSAO}

\section{Curvas ponderais em gestantes normais}

Com a finalidade de diagnosticar o estado nutricional desta população em estudo, foram construídas curvas ponderais de gestantes normais segundo a estatura, como foi proposto por Siqueira e col. ${ }^{5}$. Usando o mesmo critério, além das curvas para alturas de $1,49 \mathrm{~m}$ e menos, 1,50 a $1,54 \mathrm{~m}$; 1,55 a $1,59 \mathrm{~m}$, foram elaboradas curvas para altura de $1,60 \mathrm{~m}$ a $1,64 \mathrm{~m} ; 1,65 \mathrm{~m}$ e mais, visto que havia um grande número de mulheres com $1,65 \mathrm{~m}$ e mais de altura. As Figs. 1, 2, 3, 4 e 5 mostram as curvas ponderais com seus limites inferior e superior (média \pm um desvio-padrão), para os cinco grupos de gestantes. As gestantes cujos pesos estavam situados entre os limites superior e inferior foram consideradas magras, ou subnutridas, as que estavam acima do limite superior obesas.

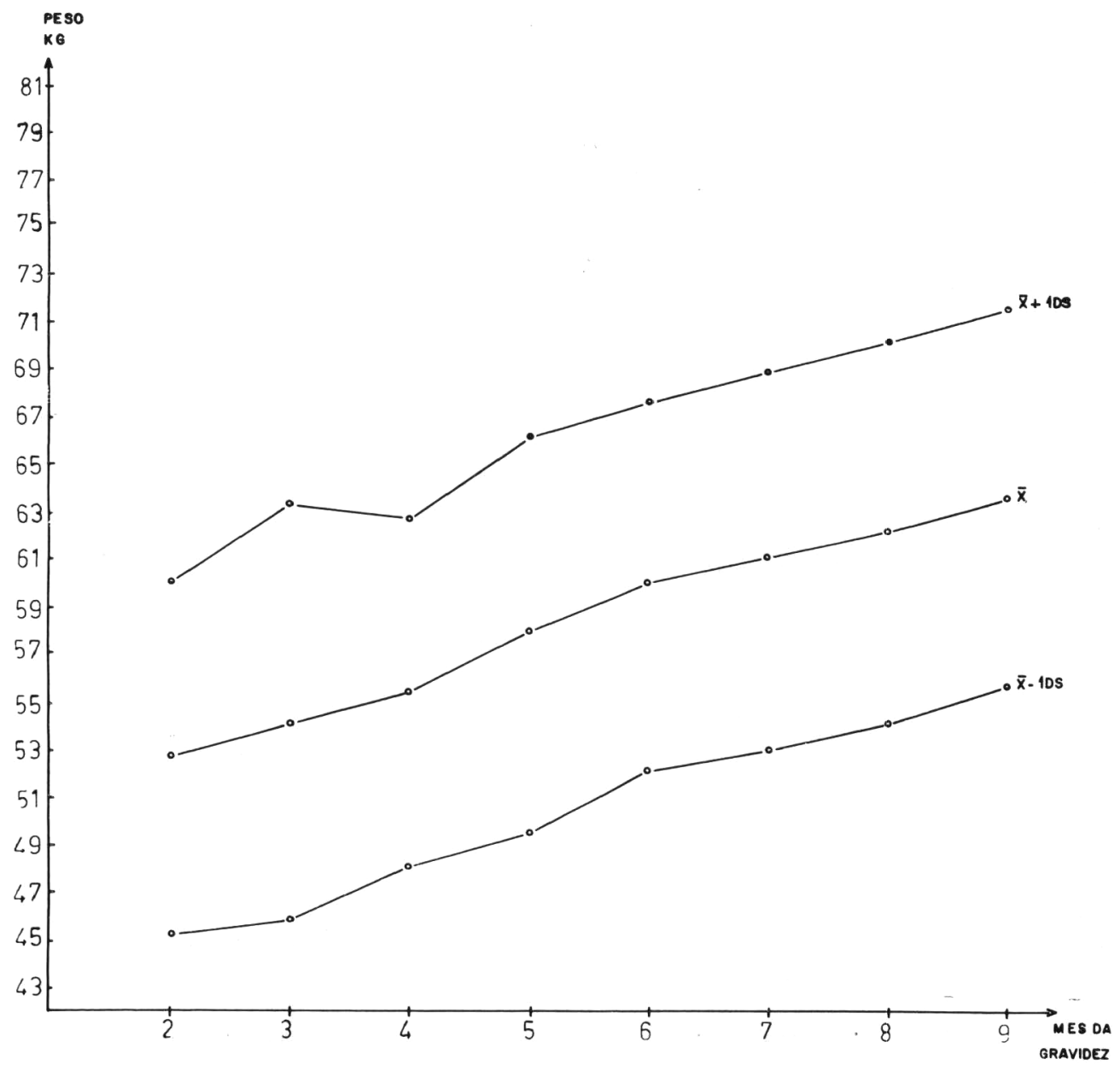

Fig. 2 - Curva ponderal para gestantes de 1,50 a $1,54 \mathrm{~m}$. 
SIQUEIRA, A. A. F. de et al. - A utilização de uma curva ponderal de gestantes normais no diagnóstico de desnutrição intra-uterina. Rev. Saúde pribl., S. Pauio, 9:495-506, 1975.

Uma vez construídas as curvas, os pesos de cada gestante foram aplicados a elas, obtendo-se os seguintes resultados: das 1.347 gestantes, 234, estavam abaixo do limite inferior, sendo consideradas magras; 894 entre os limites inferior e superior, que foram consideradas normais e 210 acima do limite superior, portanto, consideradas obesas. 0 peso médio ao nascer dos recém-nascidos das mulheres magras foi inferior ao das normais e este das obesas, sendo das magras o peso médio do recém-nascido de $2.973 \mathrm{~g}$, das normais $3.170 \mathrm{~g}$ e das obesas $3.353 \mathrm{~g}$.
O fato do peso médio dos recém-nascidos das mulheres magras (ou subnutridas) ser inferior ao das normais e este ao das obesas, mostra uma nítida relação entre estado nutricional materno (dado pelo peso) e peso do recém-nascido, uma vez que os grupos de mulheres foram baseados na estatura

Estes resultados mostram a aplicabilidade desta curva no diagnóstico precoce e na correção da subnutrição e obesidade maternas que podem ser controladas durante o pré-natal, com programas médico-nutricionais adequados. Ainda per-

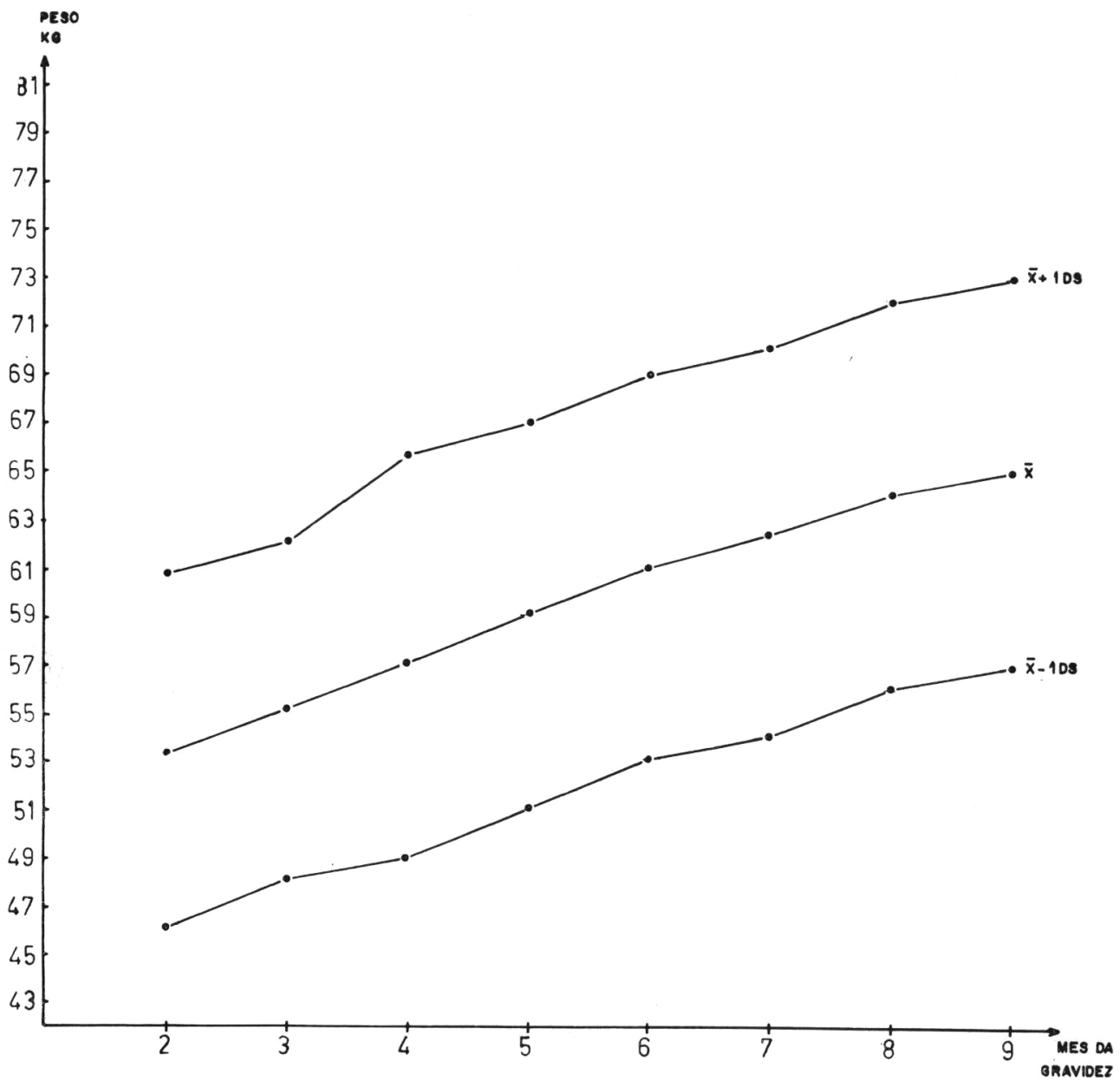

Fig, 3 - Curva ponderal para gestantes de 1,55 a $1,59 \mathrm{~m}$. 
SIQUEIRA, A. A. F. de et al. - A utillzação de uma curva ponderal de gestantes normais no diagnóstico de desnutrição intra-uterina. Rev. Saúde públ., S. Paulo, 9:495-506, 1975.

mite diagnosticar durante a gravidez as gestantes que tendem a cair num dos extremos das curvas e que devem ter uma atenção pré-natal mais controlada.

Influência do estado nutricional materno sobre o peso do recém-nascido

Sabendo que a idade gestacional é uma das variáveis que mais influi no peso do recém-nascido, estes foram separados em três grupos, os prematuros com 36 semanas gestacionais e menos, os normais com
37 a 41 semanas e os pós-termo com 42 semanas e mais.

Foi estudada a incidência de prematuridade e pós-termo. Os resultados observados encontram-se nas Tabelas 1 e 2 .

A Tabela 1 mostra que a incidência de prematuridade nas crianças de gestantes magras é duas vezes maior que as das gestantes normais e das obesas, sendo esta semelhante para as normais $e$ as obesas.

Observa-se também uma maior incidência de prematuridade nas mulheres baixas $(1,49 \mathrm{~m}$ e menos) e magras. 0 peso médio dos prematuros das mulheres ma-

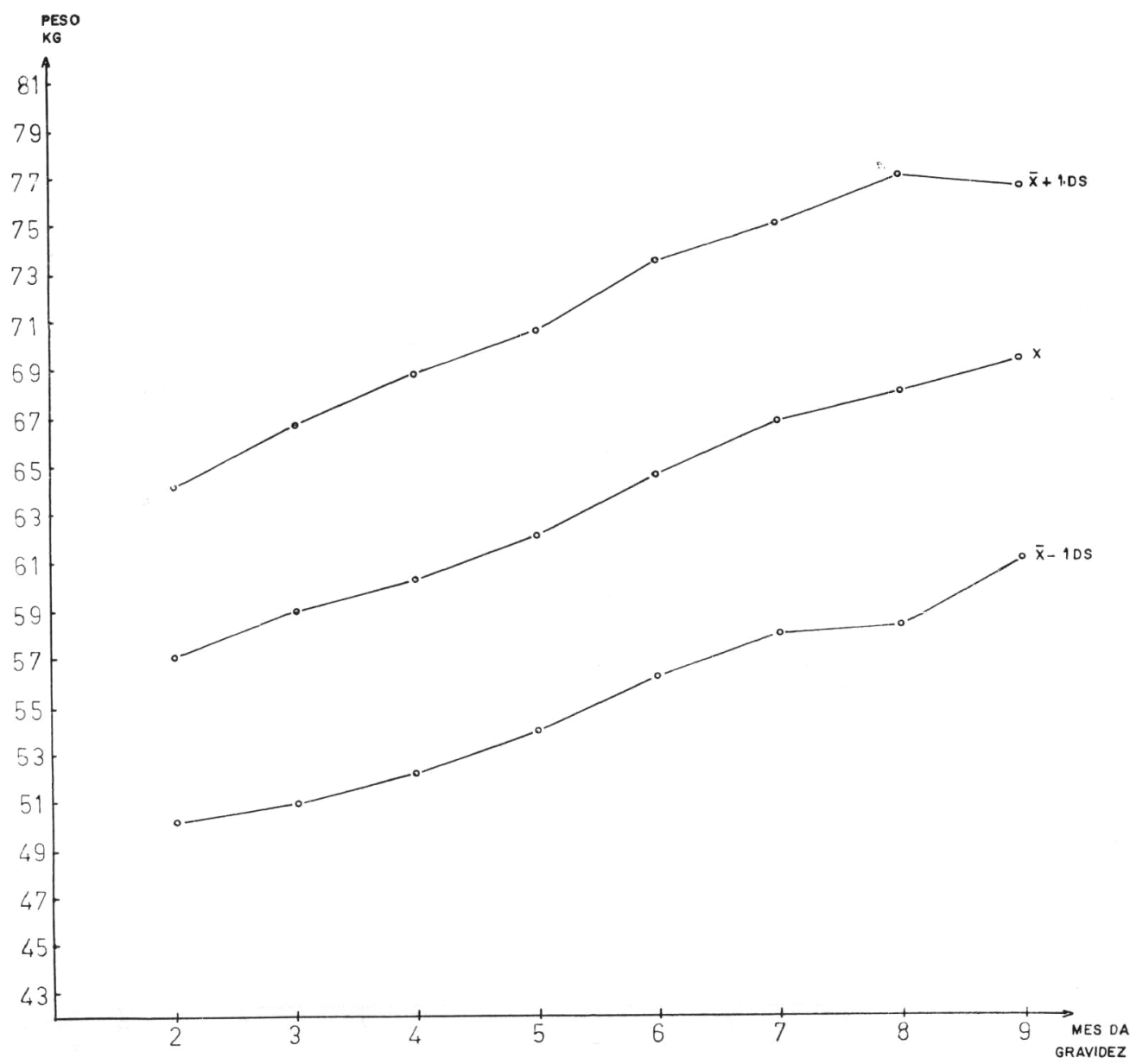

Fig. 4 - Curva ponderal para gestantes de 1,60 a $1,64 \mathrm{~m}$. 
SIQUEIRA, A. A. F, de et al, - A utilização de uma curva ponderal de gestantes normais no diagnóstico de desnutrição intra-uterina. Rev. Saúde públ., S. Paulo, 9:495-506, 1975.

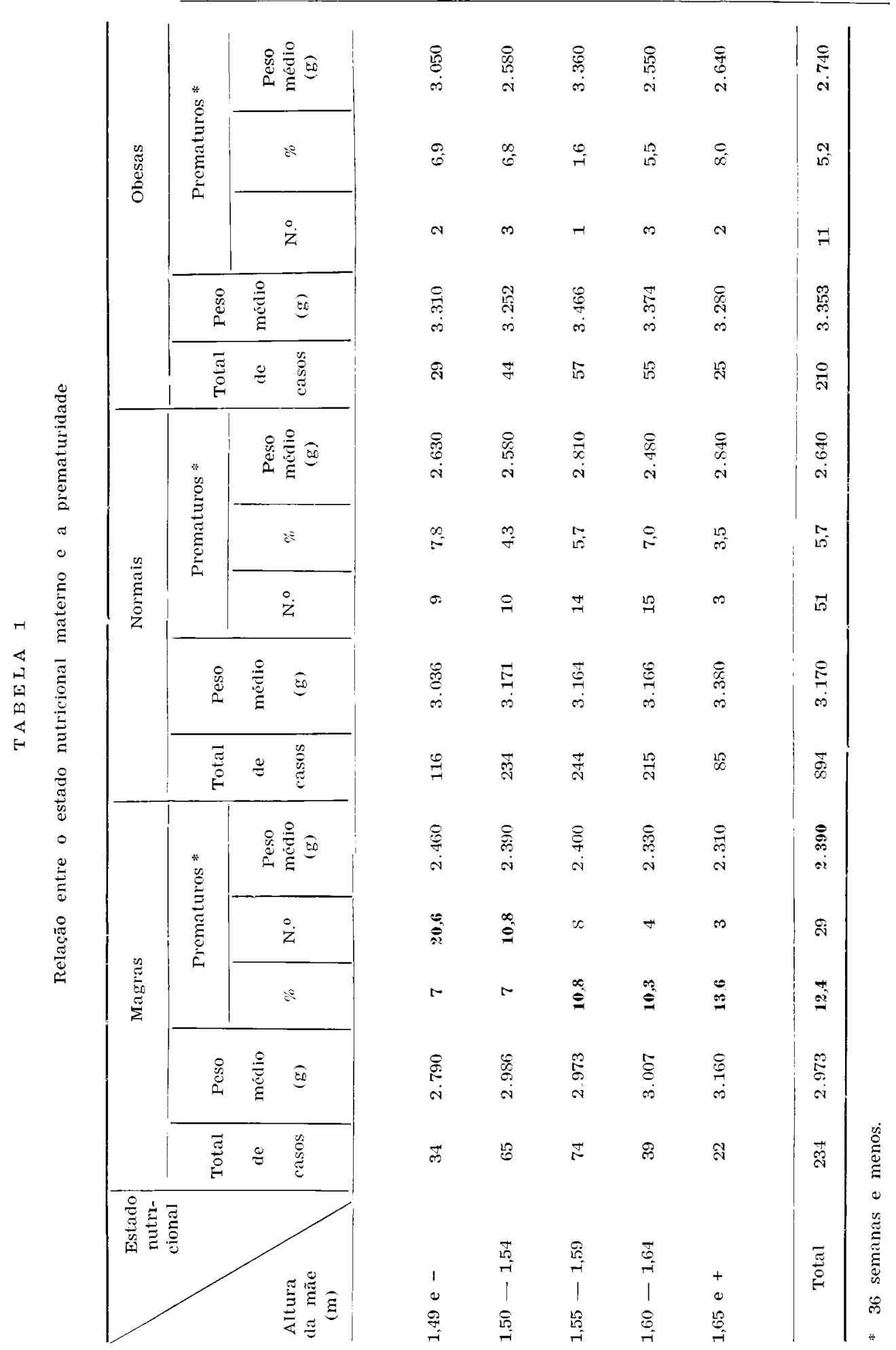


SIQUEIRA, A. A. F. de et al. -- A utilização de uma curva ponderal de gestantes normais no diagnóstico de desnutrição intra-uterina. Rev. Saúde públ., S. Paulo, 9:495-506, 1975.

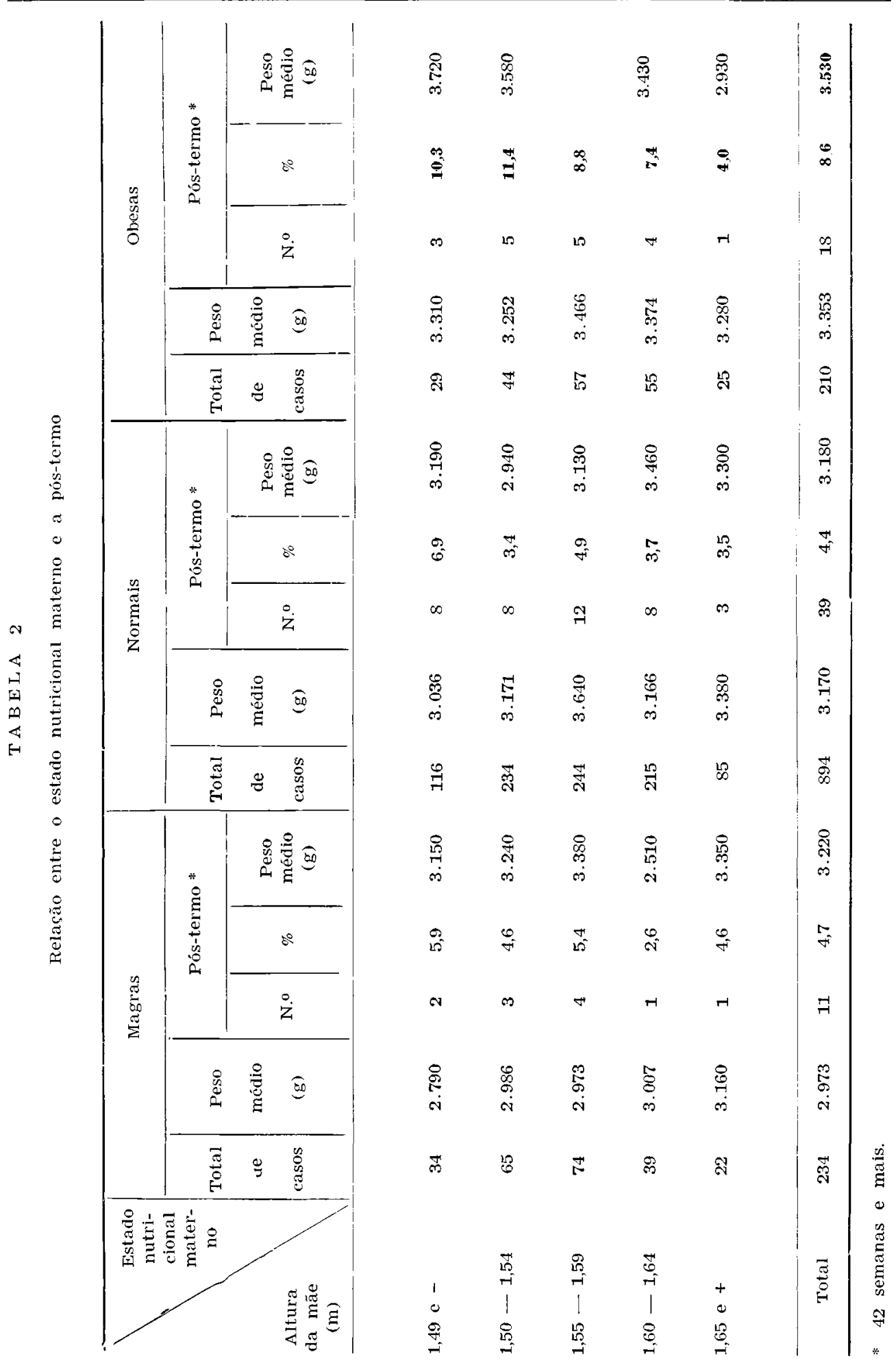


SIQUEIRA, A. A. F. de et al. - A utilização de uma curva ponderal de gestantes normais no diagnóstico de desnutrição intra-uterina. Rev. Saúde públ., S. Paulo, :495-506, 1975.

gras é inferior ao das normais e ao das obesas, sendo menor que $2.500 \mathrm{~g}$; os dos outros 2 grupos de gestantes são superiores a $2.500 \mathrm{~g}$ e muito próximos entre si.

A Tahela 2, por sua vez, mostra que há uma incidência maior de pós-termo em gestantes obesas, sendo esta o dobro, se comparada com as das gestantes normais e magras. A incidência de pós-termo se comporta de maneira semelhante entre as grávidas magras e normais. Parece não existir uma relação entre pós-termo e altura materna. visto que esta se apresenta de maneira muito próxima em todos os grupos de altura. 0 peso médio dos pós-termo é sensivelmente maior nos recém-nascidos de gestantes obesas e semelhante nas magras e normais.

Estas tabelas mostram que existe relação entre a incidência de prematuridade e pós-termo de acordo com o estado nutricional materno, levando a alterações grandes no peso do recém-nascido.

É possível que a incidência maior de pós-termo entre as gestantes obesas seja uma decorrência da maior dificuldade de diagnóstico correto da idade gestacional nesse grupo de mulheres, pela espessura

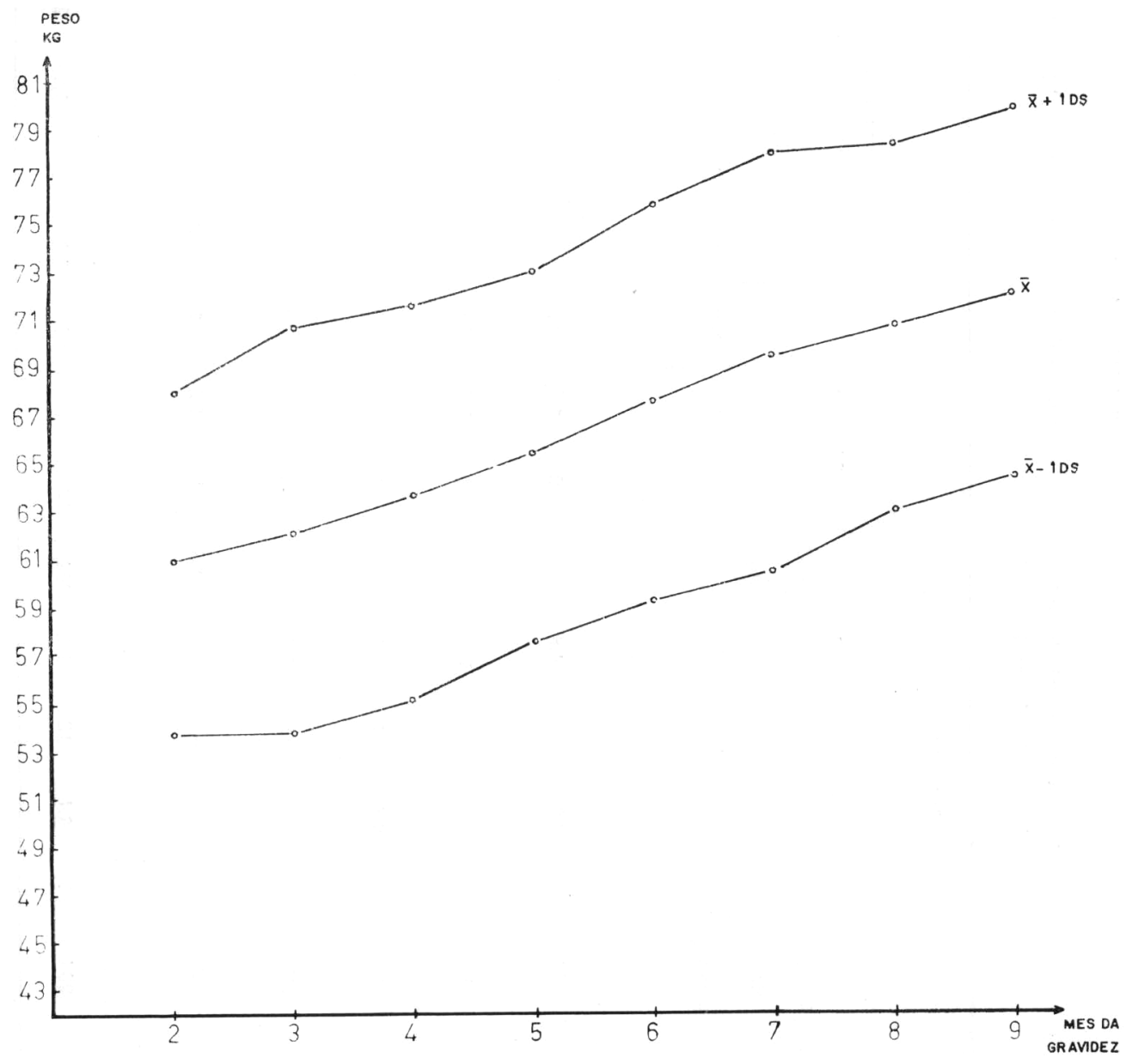

Fig 5 - Curva ponderal para gestantes de $1,65 \mathrm{~m}$ e mais. 


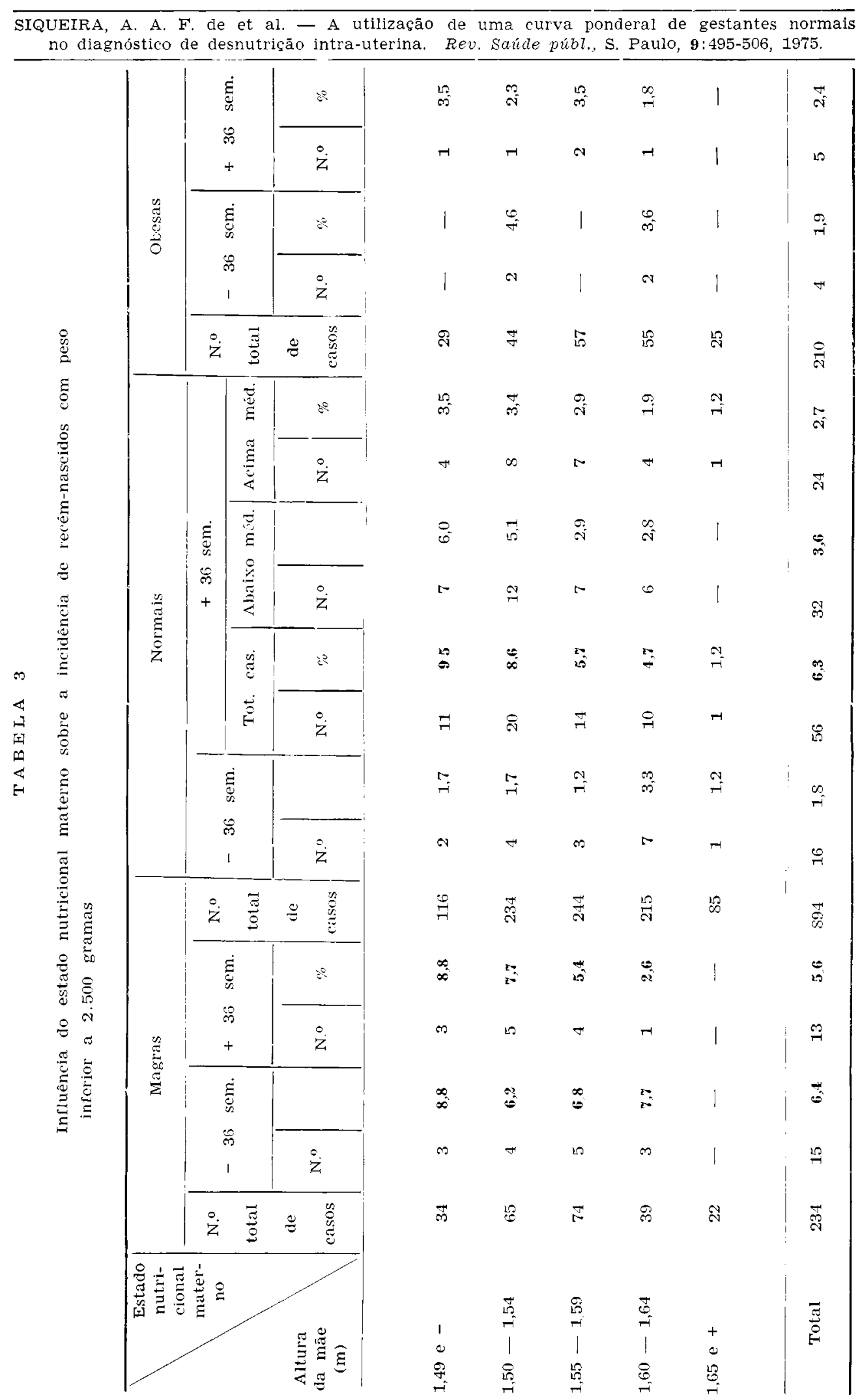


SIQUEIRA, A. A. F. de et al. - A utilização de uma curva ponderal de gestantes normais no diagnóstico de desnutrição intra-uterina. Rev. Saúde públ., S. Paulo, 9:495-506, 1975.

maior do panículo adiposo, o que prejudica a palpação uterina, um dos critérios utilizados neste trabalho para a determinação da idade gestacional.

A associação entre desnutrição materna e prematuridade ficou muito evidente.

Como hipótese de trabalho havíamos proposto que deveria haver uma incidência maior de crianças nascidas com peso igual ou inferior a $2.500 \mathrm{~g}$ no grupo de mulheres subnutridas (abaixo do limite inferior das curvas). o que realmente ocorreu, porém não somente à custa de recém-nascidos pequenos para a idade gestacional como principalmente de prematuros verdadeiros (gestaçōes de 36 semanas e menos).

Consideramos esse achado de fundamental importância no estudo de crescimento e desenrolvimento fetais. É óbvio que com um estudo antropométrico como este não se pode saber que tipo de deficiência nutricional materna (se é que realmente houve) seja a causa dessa alteração do desenvolvimento intra-uterino. Dada a importância do assunto. no entanto. a Disciplina Higiene Materna está elaborando projetos complementares de estudos bioquímicos e de dietas de uma população gestante, quando esses métodos serão cotejados com o método antropométrico aqui aplicado.

Pretendendo-se estudar a possivel relação entre o estado nutricional materno e os recém-nascidos com peso inferior a 2.500 g. foram separadas as crianças de $2.500 \mathrm{~g}$ e menos, por idade gestacional, o que possibilitou diagnosticar os prematuros (com 36 semanas ou menos) e os pequenos para idade gestacional (com 36 semanas e mais). Os resultados conseguidos encontram-se na Tabela 3 .

Nas gestantes magras a incidência de prematuridade e a de pequenos para idade gestacional são próximas. Quanto mais baixa a mulher maior a incidência de pequenos para a idade gestacional. Os prematuros de $2.500 \mathrm{~g}$ e menos têm uma incidência muito semelhante para cada gru- po de altura, fazendo exceção as gestantes com 1.65 m e mais, que não apresentaram nenhum caso tanto de prematuros como de pequenos para a idade gestacional.

É interessante salientar que nas mulheres normais observou-se uma incidência três vezes maior de pequenos para a idade gestacional que a de prematuros. Ao se estudar o ganho de peso destas gestantes aplicado na curva ponderal observou-se que $60 \%$ destas mulheres apresentaram pesos abaixo da média (próximos ao limite inferior) ou ganho de peso negativo. Isso permitiu concluir que estas mulheres são desnutridas em potencial, e por isto, os recém-nascidos apresentam um maior risco de desnutrição intra-uterina.

Da mesma forma que as magras, as gestantes normais que têm seu peso abaixo da média apresentam maior incidência de pequenos para idade gestacional no grupo de mulheres baixas (1.49 $\mathrm{m}$ e menos), decrescendo estes valores de acordo com o aumento de estatura.

As gestantes obesas possuem incidência muito baixa tanto de prematuridade como de pequenos para a idade gestacional, sendo estas muito próximas.

Este estudo possibilitou verificar a aplicabilidade das curvas ponderais de gestantes normais no diagnóstico do estado nutricional materno e avaliar o risco de desnutrição intra-uterina e prematuridade a que o concepto está sujeito.

\section{CONCLUSÕES}

1. A identificação da desnutrição materna e da desnutrição intra-uterina, são passiveis de serem feitas a nível de pré-natal, principalmente se forem aplicadas as "Curvas Ponderais de Gestantes Normais". por nós elaborada e que são de fácil manuseio.

2. Com a aplicação da curva foi diagnosticado o estado nutricional das gestantes, classificadas em 3 grupos: magras, normais e obesas. Verificou-se que o pe- 
SIQUEIRA, A. A. F. de et al. - A utllização de uma curva ponderal de gestantes normais no điagnóstico de desnutrição intra-uterina. Rev. Saúde públ., S. Paulo, 9:495-506, 1975.

so dos recém-nascidos das gestantes magras era inferior ao das normais e este ao das obesas.

3. A incidência da prematuridade foi duas vezes maior entre os recém-nascidos das mulheres magras que entre as demais. A incidência de pós-termo em gestantes obesas foi o dobro das demais, mostrando uma relação entre estado nutricional materno e idade gestacional.

\begin{abstract}
RSBU-B/287
Siqueira, A. A. F. de et al. - [On the use of a normal pregnant women's weight curve in the diagnosis of intra-uterine malnutrition]. Rev. Saude públ., S. Paulo, 9:495-506, 1975.

SUMMARY: With the purpose of studying the influence of the maternal nutritional condition on the newborn's weight, the authors applied the "Normal pregnant women's weight curves" to 1,347 patients. The use of these curves allowed identification of the maternal nutritional condition. These pregnant women were divided into three groups: 234 undernourished, 894 normal and 210 obese women. The newborns' weight at birth were compared in each group of pregnant women and the results showed that, the incidence of pregmaturity was twice as large in the undernourished pregnant women's newborns, as was also the incidence of post-term in obese pregnant women. The greatest incidence of children small for their gestational age was among undernourished pregnant women and in normal pregnant women whose weight gain was under the average values of the "Normal pregnant women's weight curves".
\end{abstract}

UNITERMS: Birth weight, maternal nutritional condition. Prematurity.

\title{
REFERENCIAS BIBLIOGRAFICAS
}

1. CIARI Jr., C. - Curva ponderal de gestantes normais. Folha méd, Rio de Janeiro, 68:141-6, 1974.

2. GRUENWALD, P. - Growth of the human fetus. I. Normal growth and its variation. Amer. J. Obstet. Gynec, 94: $1112-9,1966$.

3. HYTTEN, F. E. \& LEITCH, I. - The physiology of human pregnancy. Oxford, Blackwell, 1964.

4. JELLIFFE, D. B. - Evaluación del estado de nutricion de la comunidad (con especial referência a las en- cuestas en las regiones en desarollo). Ginebra, Organización Mundial de la Salud, 1968. (Serie de Monografias, 53).

5. SIqUEIRA, A. A. F. de et al. - Influencia da altura e ganho de peso maternos e de idade gestacional sobre - peso do recém-nascido: estudo de 3 grupos de gestantes normais. Rev. saúde públ., S. Paulo, 9:331-42, 1975.

Recebido para publicação em 05/09/1975 Aprovado para publicaça em 22/09/1975 